\title{
ATRIBUTOS HISTOLÓGICOS RELACIONADOS CON DIGESTIBILIDAD EN Bouteloua curtipendula (MICHX.) TORR. DE MÉXICO
}

\author{
DIGESTIBILITY-RELATED HISTOLOGICAL ATTRIBUTES IN Bouteloua \\ curtipendula (MICHX.) TORR. OF MEXICO
}

\begin{abstract}
Álvaro Bernal-Flores ${ }^{1,2}$, Adrián R. Quero-Carrillo², Hilda A. Zavaleta-Mancera²*, Paulino Pérez-Rodríguez ${ }^{2}$, Jorge Valdez-Carrasco ${ }^{2}$ y Ma. Esther Ortega-Cerrilla ${ }^{2}$
\end{abstract}

\begin{abstract}
'Semillas Papalotla S.A. de C.V., Centro de Investigación en Pastos Tropicales. Santa Elena del Tule, Puerto Escondido, Oaxaca. ${ }^{2}$ Campus Montecillo. Colegio de Postgraduados. km 36.5 Carr. México-Texcoco. 56230, Montecillo, Estado de México.

*Autor para correspondencia (arazavaleta@colpos.mx)
\end{abstract}

\section{RESUMEN}

Pasto banderita (Bouteloua curtipendula (Michx.) Torr.) es un forraje $\mathrm{C}_{4}$ de gran diversidad genética, adaptado a regiones áridas y semiáridas del norte de México y sur de los E.E.U.U. En este estudio se evaluaron los atributos histológicos asociados con digestibilidad y lignificación en 30 genotipos de $B$. curtipendula de México para conocer su potencial forrajero. Con un diseño completamente al azar se muestrearon hojas verdes maduras a los 65 d de rebrote de una colección viva. El tejido foliar se fijó y procesó para cortes transversales $(15 \mu \mathrm{m})$ de parafina, teñidos con safranina- 0 y verde rápido FCF. El tejido lignificado se detectó con fluoroglucinol y $\mathrm{HCl}$. Los atributos histológicos medidos en el área en corte transversal fueron: tejidos altamente digestibles, TAD (mesófilo, células buliformes y floema); tejidos parcialmente digestibles, TPD (epidermis, vaina del haz vascular y extensión no lignificada de la vaina del haz vascular), y tejidos no digestibles T no-D (xilema y extensión lignificada de la vaina del haz vascular) en nervaduras principales (NP) y secundarias (NS). Los datos se analizaron con un análisis de componentes principales (ACP) y después los genotipos se agruparon por atributo histológico con el método jerárquico basado en distancias Euclidianas; las medias de grupos se compararon con Tukey $(P \leq 0.05)$. Tres componentes principales (CP) explicaron más del $90 \%$ de la variabilidad anatómica y las NS presentaron $21 \%$ más área de TAD que las NP. El mesófilo (28\%), las células buliformes $(23 \%)$ y la vaina del haz vascular $(20 \%)$ ocuparon más del $70 \%$ del área foliar total. En promedio la hoja de B. curtipendula está compuesta por $58 \%$ de TAD, $33 \%$ de TPD y $9 \%$ de T no-D. El grupo con mayores valores de TAD y TPD incluyó a los genotipos G55, G17, G18, G16, G48 y G64, pero el G16 mostró los mejores atributos forrajeros ( $56.8 \%$ de TAD y $5.3 \%$ tejido lignificado).

Palabras clave: Anatomía foliar, forraje, pasto banderita, lignina, recursos genéticos.

\section{SUMMARY}

Sideoats grama (Bouteloua curtipendula (Michx.) Torr.) is a $\mathrm{C}_{4}$ forage species of great genetic diversity, adapted to arid and semi-arid regions of Northern Mexico and Southern U.S.A. In this study the histological attributes associated with digestibility and lignification were evaluated in 30 genotypes of $B$. curtipendula of Mexico to know their forage potential. A completely randomized design was used to sample mature green leaves at $65 \mathrm{~d}$ of regrowth from a live collection. Leaf tissue was fixed and processed for paraffin transversal sections $(15 \mu \mathrm{m})$ and stained with safranin-0 and fast green FCF. Lignified tissue was detected with phloroglucinol and $\mathrm{HCl}$. The histological attributes measured in the cross sectional area were: highly digestible tissues, TAD (mesophyll, bulliform cells and phloem); partially digestible tissues, TPD (epidermis, vascular bundle sheath and the non-lignified extension of the vascular bundle sheath), and not digestible tissues, T non-D (xylem and the lignified extension of the vascular bundle sheath) in principal (NP) and secondary ribs (NS). Data were analyzed with Principal Component Analysis (ACP) and then the genotypes were grouped by histological attribute, using the hierarchical clustering method based on Euclidean distances; the group means were compared with Tukey's test $(P \leq 0.05)$. Three principal components $(C P)$ explained more than $90 \%$ of the anatomical variability, and NS showed $21 \%$ more area of TAD than NP. The mesophyll (28\%), the bulliform cell ( $23 \%$ ) and the bundle sheath $(20 \%)$ occupied more than the $70 \%$ of the total leaf area On average, the leaf of $B$. curtipendula is composed by $58 \%$ of TAD, $33 \%$ TPD and $9 \%$ of T non-D. The group with higher values of TAD and TPD included genotypes G55, G17, G18, G16, G48 and G64, but G16 genotype showed the best forage attributes ( $56.8 \%$ of TAD and $5.3 \%$ of lignified tissue).

Index words: Leaf anatomy, forage, sideoats grama, lignin, genetic resources.

\section{INTRODUCCIÓN}

Bouteloua curtipendula (Michx.) Torr. es una especie forrajera $\mathrm{C}_{4}$ perenne y nativa de México y EE.UU. genotipos diploides, poliploides y aneuploides $(2 n=2 x$ a $12 x=20$ a 120 o más) (Barkworth et al., 2007a y b; Gould, 1979), Esta especie está adaptada a zonas áridas y semiáridas, es tolerante al pastoreo, a la sequía y a la salinidad; tiene una digestibilidad del $67 \%$ y un contenido de proteína de 10.3 \% (Craigh et al., 2001; González et al., 2010; Pitman y Jaynes, 1980; USDA, 2011). Por sus atributos forrajeros y su naturaleza perenne, puede llegar alcanzar producciones de $3700 \mathrm{~kg} \mathrm{MS} \mathrm{ha}^{-1}$ por lo que se considera con potencial pastoril en las zonas áridas de México (Craigh et al., 2001; USDA, 2011). La amplia variabilidad genética inter e intra específica en los pastos forrajeros es de gran interés para el mejoramiento genético, donde la estructura anatómica foliar es un aspecto de gran importancia para predecir el valor nutritivo (Akin y Burdick, 1975; Arellano-Cueto et al., 2017) en parte porque las características anatómicas 
están relacionadas con atributos forrajeros como la tasa de digestibilidad, el valor nutricional, la digestión de fibra y el grosor de la pared celular (Jung y Allen, 1995; Wilson y Mertens, 1995).

La epidermis, esclerénquima, parénquima y mesófilo constituyen los principales tejidos relacionados con la calidad forrajera en pastos $\mathrm{C}_{4}$ (Echenique et al., 2008), el mesófilo se caracteriza por ser un tejido altamente digestible; ahí se encuentra $70 \%$ de la proteína contenida en cloroplastos (Makino y Osmond, 1991). El mesófilo y floema son altamente degradables, la epidermis y la vaina del haz vascular se degradan parcialmente; y el esclerénquima y los vasos del xilema, altos en lignina, son no degradables (Akin, 1989; Buxton y Redfearn, 1997). El principal tejido determinante en la digestibilidad de gramíneas $\mathrm{C}_{3}$ y $\mathrm{C}_{4}$ es la abundancia de mesófilo (Buxton y Redfearn, 1997) aunque el nivel de ploidía y la ruta fotosintética también son determinantes, (Warner y Edwards, 1989).

La detección histoquímica de lignina ha permitido conocer de manera detallada la localización y abundancia det tejido lignificado (Sokupová y Albrechtová, 2003). La cuantificación histoquímica de lignina y la cuantificación química presenta altas correlaciones (de 0.90 a 0.96) (Soukupová et al., 2001; Soukupová y Albrechtová, 2003). La acumulación de lignina en los tejidos de gramíneas aumenta con la edad y senescencia; el ácido ferúlico se deposita en estadios juveniles, y el ácido p-cumárico se deposita durante todo el desarrollo (Casler y Hatfield, 2006). Diferencias en la proporción de los monolignoles siringil y guaicil, encontradas en xilema y esclerénquima están relacionadas con el grado de resistencia a la degradación ruminal (Chamorro et al., 2005a). Polímeros de lignina con mayor cantidad de unidades siringil son más colonizados por microrganismos del rumen que aquellos polímeros con mayor cantidad de unidades guaiacílicas (Chamorro et al., 2005b). Así, para un programa de mejoramiento genético, el análisis de la estructura anatómica y tejidos lignificados son indicadores confiables de la calidad de los forrajes. El objetivo del presente estudio fue cuantificar los tejidos altamente degradables (TAD), parcialmente degradable (TPD) y no degradables (T no-D) y la cantidad relativa de lignina de 30 genotipos de Bouteloua curtipendula (Michx.) Torr., para seleccionar genotipos con mejores características forrajeras.

\section{MATERIALES Y MÉTODOS}

\section{Origen del material biológico y diseño experimental}

Los 30 genotipos estudiados fueron obtenidos de una colección viva de Bouteloua curtipendula (Michx.) Torr., ubicada en el campus San Luis Potosí del Colegio de Post- graduados (Cuadro 1). Las plantas fueron sembradas a distancia de $50 \times 50 \mathrm{~cm}$ entre hileras y plantas, bajo condiciones de temporal. Las plantas se homogenizaron con un corte a $5 \mathrm{~cm}$ arriba del suelo, previo al periodo de lluvias y en cada una de tres plantas por genotipo se marcaron tres tallos con un aro de color. Se utilizó un diseño experimental completamente al azar con tres repeticiones correspondientes a tres hojas verdes con lígula expuesta. El muestreo se realizó a los 65 d de rebrote y de cada hoja se obtuvieron tres submuestras $(n=9)$. En cada submuestra se midieron seis secciones de tejido correspondientes a tres nervaduras principales (NP) y tres nervaduras secundarias (NS).

\section{Variables de estudio}

Los atributos histológicos estudiados y asociados a digestibilidad fueron clasificados según Akin y Burdick (1975), el área $\left(\mathrm{mm}^{2}\right)$ en corte transversal de: tejidos altamente digestibles (TAD) [mesófilo (M) células buliformes (CB) y floema (F)]; tejidos parcialmente digestibles (TPD) [vaina del haz vascular $(\mathrm{VH})$, epidermis (E) y extensión no lignificada de la vaina del haz (EVH-nl) ]; y tejidos no digestibles ( $T$ no-D) [xilema $(X)$ y extensión lignificada de la vaina del haz $(E V H-I)]$. Estos tejidos se midieron en tres nervaduras principales (NP) y tres nervaduras secundarias (NS) por hoja $(n=18)($ Figura 1$)$.

\section{Procesamiento histológico}

Un fragmento de $1 \mathrm{~cm}$ de la parte media de cada hoja se fijó en solución FAA (10 \% formaldehído a 37 \%, 5 \% ácido acético glacial, $50 \%$ de etanol y $35 \%$ agua) durante 48 h. Los fragmentos se cortaron transversalmente; la mitad de los fragmentos se procesó para la obtención de cortes transversales $(15 \mu \mathrm{m})$ de parafina teñidos con safranina O y verde rápido FCF (Ruzin, 1999; Zavaleta-Mancera et al., 2003), de la otra mitad de tejidos fijados se obtuvieron cortes transversales $(50 \mu \mathrm{m})$, los cuales fueron teñidos con fluoroglucinol (2\% en etanol) y ácido clorhídrico $50 \%$ para la detección de lignina (Wittenberg et al., 1993). Esta técnica detecta en rojo las unidades coniferilos y siringilos en medio ácido (Gahan et al., 1984).

Los cortes se observaron y fotografiaron con un fotomi-

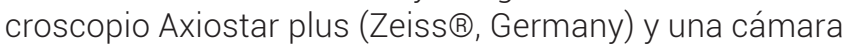
MotiCAM 2000 (Motic ${ }^{\circledR}$, Hong Kong). Para los atributos histológicos, las imágenes fueron segmentadas con el software GIMP 2.8 (GIMP Documentation Team, 2010) (Figura 1B) y el área fue calculada con el software Image Tool para Windows 3.00 (http:// ddsdx.uthscsa.edu/dig/ itdesc.html). El área de tejido lignificado fue seleccionada con la herramienta 'Treshold' del software Image Tool. Las áreas fueron calculadas en términos relativos (área 
Cuadro 1. Procedencia de los 30 genotipos evaluados de Bouteloua curtipendula (Michx.) Torr.

\begin{tabular}{|c|c|c|}
\hline Genotipos & Sitio de procedencia & Coordenadas \\
\hline G01, G04, & km 91.02 Carr. Saltillo-Piedras Negras & $26^{\circ} 05^{\prime} 37.6^{\prime \prime} \mathrm{N} ; 101^{\circ} 35^{\prime} 36.0^{\prime \prime} \mathrm{O}$ \\
\hline G05, G06, & km 72.15 Carr. Saltillo-Piedras Negras & $25^{\circ} 93^{\prime} 96.0^{\prime \prime} \mathrm{N} ; 101^{\circ} 22^{\prime} 92.0^{\prime \prime} \mathrm{O}$ \\
\hline G08, G09, G10 & km 22 Carr. Saltillo-Piedras Negras & $25^{\circ} 57^{\prime} 65.0^{\prime \prime} \mathrm{N} ; 101^{\circ} 59^{\prime} 86.0^{\prime \prime} \mathrm{O}$ \\
\hline G13, G14, G15, G16 & km 336.8 Carr. Saltillo-Zacatecas & $25^{\circ} 33^{\prime} 57.4^{\prime \prime} \mathrm{N} ; 101^{\circ} 05^{\prime} 08.2^{\prime \prime} \mathrm{O}$ \\
\hline G17, G18, & km 308.9 Carr. Saltillo-Zacatecas & $23^{\circ} 80^{\prime} 29.3^{\prime \prime} \mathrm{N} ; 102^{\circ} 08^{\prime} 05.5^{\prime \prime} \mathrm{O}$ \\
\hline G19, G20 & km 298.6 Carr. Saltillo-Zacatecas & $23^{\circ} 22^{\prime} 10.6^{\prime}$ 'N; $102^{\circ} 05^{\prime} 25.9^{\prime \prime} \mathrm{O}$ \\
\hline G27, & km 285.1 Carr. Saltillo-Zacatecas & $23^{\circ} 25^{\prime} 19.2^{\prime \prime} \mathrm{N} ; 102^{\circ} 13^{\prime} 08.3^{\prime \prime} \mathrm{O}$ \\
\hline G37, G38, G40 & km Carr. Saltillo-Zacatecas & $24^{\circ} 43^{\prime} 31.4^{\prime \prime} \mathrm{N} ; 101^{\circ} 12^{\prime} 58.0^{\prime \prime} \mathrm{O}$ \\
\hline G44, G45, G47 & km 285.26 Carr. Saltillo-Zacatecas & $24^{\circ} 18^{\prime} 28.3^{\prime \prime} \mathrm{N} ; 101^{\circ} 24^{\prime} 30.4^{\prime \prime} \mathrm{O}$ \\
\hline G48 & km 261 Carr. Saltillo-Zacatecas & $24^{\circ} 31^{\prime} 12.3^{\prime \prime} \mathrm{N} ; 101^{\circ} 22^{\prime} 08.7^{\prime \prime} \mathrm{O}$ \\
\hline G50, G55 & km 236 Carr. Saltillo-Zacatecas & $24^{\circ} 40^{\prime} 46.6^{\prime \prime} \mathrm{N} ; 101^{\circ} 16^{\prime} 42,2^{\prime \prime} \mathrm{O}$ \\
\hline G64 & km 179.4 Carr. Saltillo-Zacatecas & $24^{\circ} 07^{\prime} 09.6^{\prime \prime} \mathrm{N} ; 1^{\circ} 01^{\circ} 31^{\prime} 23.6^{\prime \prime} \mathrm{O}$ \\
\hline G97 & km 38.97 Carr. San Luis Potosí-Zacatecas & $22^{\circ} 20^{\prime} 28.9^{\prime \prime} \mathrm{N} ; 101^{\circ} 14^{\prime} 43.3^{\prime \prime} \mathrm{O}$ \\
\hline G114 & km 122 Carr. San Luis Potosí-Zacatecas & $22^{\circ} 65^{\prime} 20.2^{\prime \prime} \mathrm{N} ; 101^{\circ} 94^{\prime} 82.5^{\prime \prime} \mathrm{O}$ \\
\hline G146 & km 125 Carr. Oaxaca-Ciudad de México & $17^{\circ} 40^{\prime} 54.1^{\prime \prime} \mathrm{N} ; 97^{\circ} 34^{\prime} 58.0^{\prime \prime} \mathrm{O}$ \\
\hline G303 & km 221 Carr. 45 Durango-Parral & $25^{\circ} 64^{\prime} 01.1^{\prime \prime} \mathrm{N} ; 104^{\circ} 65^{\prime} 89.8^{\prime \prime} \mathrm{O}$ \\
\hline
\end{tabular}
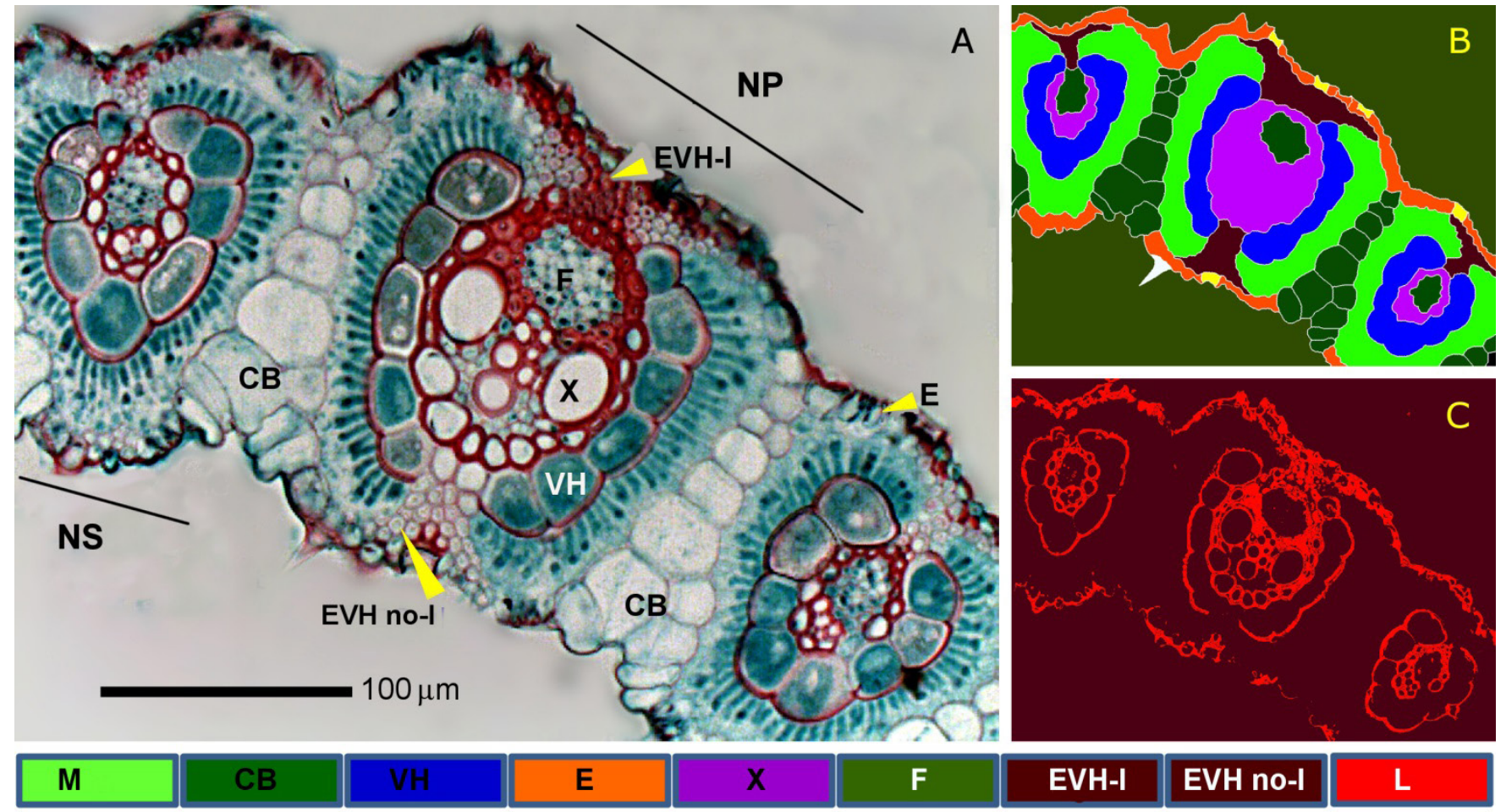

E

$\mathbf{F}$

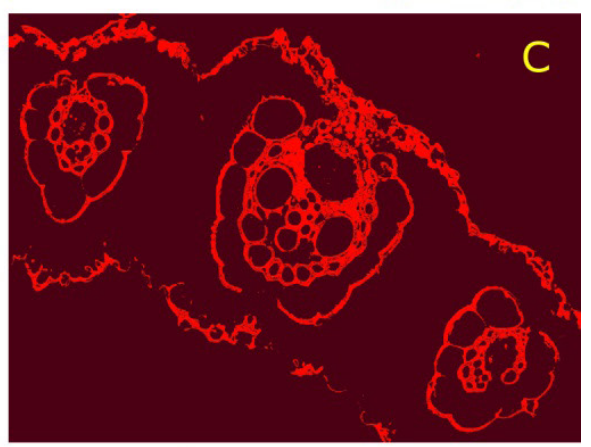

Figura 1. Corte transversal de la hoja de Bouteloua curtipendula y procesamiento de imágenes. A) Tinción con safranina-0 y verde rápido FCF. B) Segmentación de una imagen con el software GIMP. 2.8. C) La presencia de lignina se muestra en rojo, al aplicar la herramienta "threshold" del software Image Tool. NP. nervadura principal; NS: nervadura secundaria; M: mesófilo; CB: células buliformes; VH: vaina del haz vascular; E: epidermis; X: xilema; F: floema; EVH-I: extensión lignificada de la vaina del haz; EVH no-I: extensión no lignificada de la vaina del haz vascular. 
lignificada/área foliar total) y expresadas en porcentaje (Figura 1C).

\section{Análisis estadístico}

Con los datos se realizó un análisis multivariado MANOVA, ANOVA, y un análisis de componentes principales. Los datos de tejido lignificado se transformaron mediante la función arco seno y después se analizaron con un modelo mixto. Los genotipos se agruparon por atributo histológico con el método jerárquico basado en distancias Euclidianas y como criterio de proximidad entre grupos, el del vecino más cercano. Las medias de los grupos se compararon con la prueba de Tukey $(P \leq 0.05)$ usando el programa SAS (SAS Institute, 2004). Para verificar la calidad del agrupamiento se calculó la partición basada en medioides, donde los anchos de silueta cercanos a 1 indican un buen agrupamiento y valores negativos indican que un genotipo está mal clasificado (Kaufman y Rousseuw, 2005). Las comparaciones de medias entre grupos fueron realizadas mediante DHS $(P \leq 0.05)$. Los datos se analizaron con el programa R versión 3.2.2 (R Core Team, 2015).

\section{RESULTADOS Y DISCUSIÓN}

\section{Análisis de componentes principales}

Este análisis aglomeró los genotipos en dos grupos ( $\mathrm{Fi}$ gura 2A), lo que denota diferencias anatómicas entre áreas para los tipos de tejido evaluados. El grupo I fue homogéneo y compacto, integrado por genotipos que mostraron la mayor área en sus tejidos; el grupo II presentó mayor variabilidad y agrupó a un mayor número de genotipos con áreas de tejido más reducidas. Los tres primeros componentes principales explicaron $91 \%$ de la varianza total en los datos. El CP1 está mayormente asociado con la presencia de células buliformes, mientras que los CP2 y CP3 con mesófilo y vaina del haz vascular, respectivamente (Figura 2A). Tanto células buliformes como vaina del haz vascular fueron las variables que presentaron mayores varianzas y exhibieron diferencias significativas $(P \leq 0.1)$ entre genotipos. En contraste el xilema (CP5), floema (CP6), epidermis (CP7) y extensión de la vaina del haz vascular (CP8) explicaron $9 \%$ de la variación total en los datos y no presentaron diferencias significativas $(P>0.05)$ (Figura 2B). La proporción de la varianza explicada por componente fue superior a la encontrada por Morales-Nieto et al. (2006) en un estudio realizado mediante polimorfismos de la longitud de fragmentos amplificados (AFLPs), en el que dos de los componentes principales explicaron $21.8 \%$ de la variación.

En promedio, los 30 genotipos de $B$. curtipendula mostraron: $58 \%$ de TAD, $33 \%$ TPD y $9 \%$ de T no-D. En contraste, Bouteloua repens presentó valores diferentes: $56.7 \%$ TAD, $12.7 \%$ TPD y $30.5 \%$ T no-D en dos épocas de estudio (Chamorro et al., 2005b). Factores que pueden modificar estos atributos relacionados con la digestibilidad son: espesor de
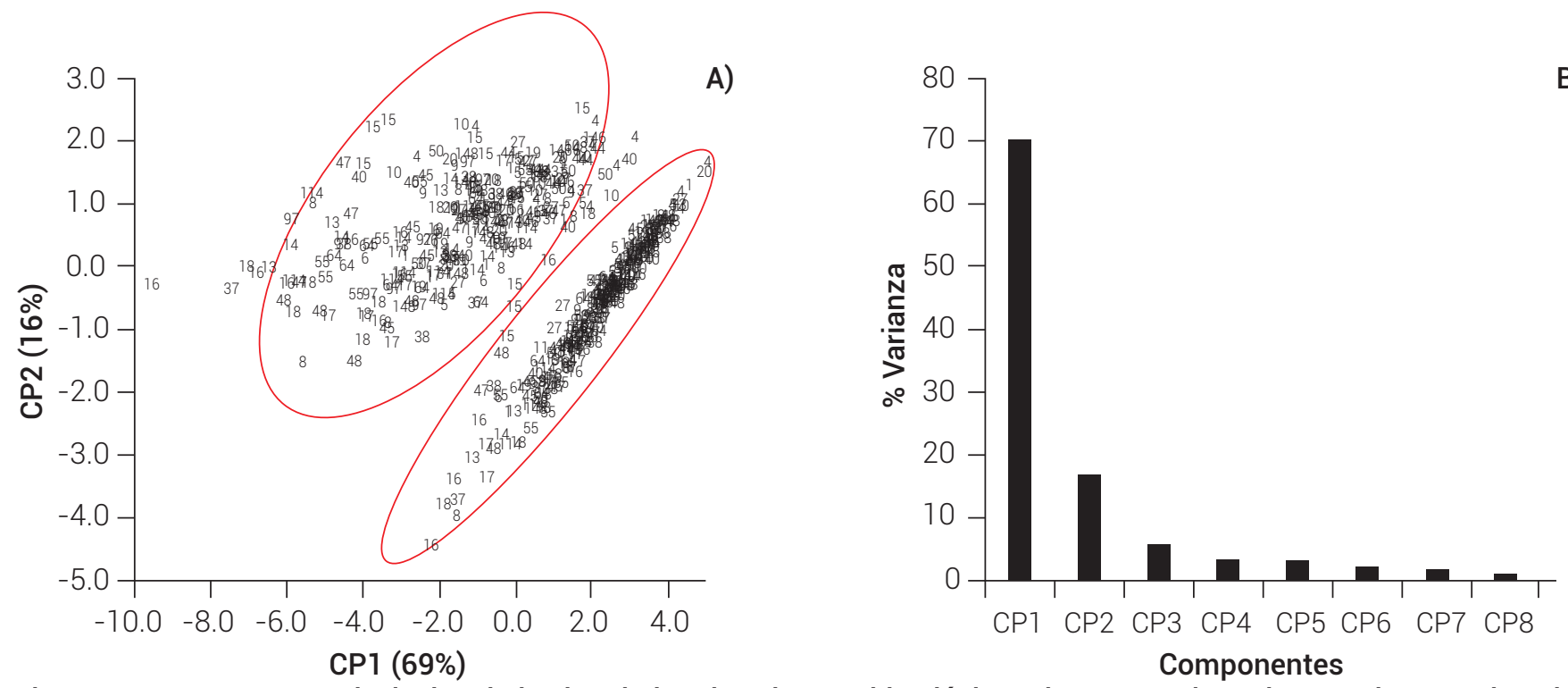

B)

Figura 2. Componentes principales derivados de los descriptores histológicos de 30 genotipos de Bouteloua curtipendula. A) Formación de grupos según CP1 y CP2; B) Proporción de la varianza explicada por los componentes. Variables de mayor influencia en los componentes: CP1: células buliformes; CP2: mesófilo; CP3: vaina del haz vascular; CP4: extensión no lignificada de la vaina del haz; CP5: xilema; CP6: floema; CP7: epidermis; CP8: extensión lignificada de la vaina del haz vascular. 
la pared celular, cantidad de mesófilo y cantidad de tejido lignificado (Campos et al., 2002). Las nervaduras principales (NP) y nervaduras secundarias (NS) presentaron diferencias estadísticas $(P<0.01)$ (Cuadro 2). En las NP la proporción total de TAD, TPD y T no-D, fue del $50.7 \%$, 32.5 $\%$ y $16.6 \%$, respectivamente, en las que se observaron las mayores diferencias con el T no-D de la NS (3\%).

Las NS presentaron gran área de mesófilo (32.9\%) y ausencia de lignificación en la extensión de la vaina, ésto se reflejó en una alta proporción de TAD (65\%). Este resultado indica que la digestibilidad de B. curtipendula está estrechamente relacionada con la densidad (No. de nervaduras $\mathrm{mm}^{-2}$ ) de las nervaduras menores (NS).

\section{Agrupamiento jerárquico}

Este método basado en distancias Euclidianas y el método del vecino más cercano separaron cinco grupos bien definidos para TAD, TPD y T no-D (Figura 3). Según las particiones basadas en medioides y el ancho de silueta (Kaufman y Rousseuw, 2005), permitió agrupar a los genotipos en cinco grupos, claramente definidos para TAD (Figura 3). El mesófilo, parénquima y células de esclerénquima mostraron gran variación en tamaño y forma, por lo que son consideradas características útiles para selección intraespecífica (Buxton y Redfearn, 1997). En el caso de B. curtipendula, las células buliformes (CP1) presentaron mayor variación que el mesófilo (CP2).

El grupo 5, integrado por los genotipos G55, G17, G18, G16, G48 y G64 fue significativamente diferente $(P \leq 0.05)$ para TAD, TPD y T no-D, en la mayoría de tejidos (Cuadro 3). En estos genotipos el mesófilo fue el tejido que mayormente contribuyó al TAD. Con base en los resultados, el área relativa del mesófilo en B. curtipendula (57.7\%) fue mayor al reportado para el pasto Bermuda (Cynodon dactylon) (43.7 \%) (Campos et al., 2002).

El mesófilo es el tejido de mayor importancia fotosintética para la planta y junto con el floema son considerados los tejidos más digestibles y de rápida degradación microbiana (Akin, 1989). Este tejido es considerado un rasgo hereditario de importancia forrajera y relevante en programas de mejoramiento genético. El mesófilo aporta del 70 al 80 $\%$ de la proteína foliar, la cual se encuentra principalmente en las proteínas de membrana del cloroplasto (LHCP2, D1, Cyt, f) y la proteína estromal; ribulosa 1,5 bifosfato carboxilasa oxigenasa (Rubisco), la cual comprende $50 \%$ de la proteína soluble de la hoja (Makino y Osmond, 1991; Wada et al., 2009). En contraste, 50 \% de los carbohidratos están contenidos en células de la vaina de los haces vasculares (Echenique et al., 2008).

De acuerdo con el análisis de agrupamiento, las mayores áreas de tejido correspondientes a los TAD fueron decisivas en la selección. Los genotipos del grupo cinco (G55, G17, G18, G16, G48 y G64) presentaron los valores más altos en TAD y TPD. Los genotipos G16, G18 y G55 fueron los que presentaron una mayor área total, pero sólo el G16 y G55 se agruparon con los genotipos de mayor área de $T A D$, los cuales tienen un mayor potencial forrajero y pueden considerarse como candidatos para mejoramiento genético (Figura 4). Carmo-Silva et al., (2009) sugieren que el tamaño de los tejidos es producto de la interacción genotipo $\times$ ambiente, lo que sugiere que la variación en la proporción de tejidos es el resultado de la adaptación del genotipo a las condiciones ambientales.

En este estudio, los 30 genotipos de B. curtipendula fueron sembrados en invernadero y estuvieron bajo las mismas condiciones de riego, temperatura e irradiación, para

Cuadro 2. Área $\left(\mu \mathrm{m}^{2} \times 10^{3}\right)$ y proporción (\%) en corte transversal de los atributos histológicos de Bouteloua curtipendula, en la nervadura central (NP) y en las nervaduras secundarias (NS) asociados con su digestibilidad.

\begin{tabular}{|c|c|c|c|c|c|c|c|c|}
\hline \multirow{2}{*}{$\begin{array}{l}\text { Tipo de } \\
\text { nervadura }\end{array}$} & \multicolumn{3}{|c|}{ Tejidos altamente digestibles (TAD) } & \multicolumn{3}{|c|}{$\begin{array}{c}\text { Tejidos parcialmente digestibles } \\
\text { (TPD) }\end{array}$} & \multicolumn{2}{|c|}{$\begin{array}{c}\text { Tejidos no } \\
\text { digestibles (T no-D) }\end{array}$} \\
\hline & M & $\mathrm{CB}$ & $\mathrm{F}$ & $\mathrm{VH}$ & E & EVH no-I & $x$ & EVH-I \\
\hline $\mathrm{NP}\left(\mu \mathrm{m}^{2} \times 10^{3}\right)$ & $6.50 \pm 0.59$ & $5.11 \pm 0.63$ & $1.71 \pm 0.21$ & $4.81 \pm 0.32$ & $2.51 \pm 0.21$ & $1.22 \pm 0.16$ & $3.44 \pm 0.41$ & $0.92 \pm 0.12$ \\
\hline NP $(\%)$ & 24.8 & 19.5 & 6.5 & 18.3 & 9.6 & 4.7 & 13.1 & 3.5 \\
\hline $\mathrm{NS}\left(\mu \mathrm{m}^{2} \times 10^{3}\right)$ & $5.42 \pm 0.52$ & $4.69 \pm 0.59$ & $0.67 \pm 0.09$ & $3.62 \pm 0.35$ & $1.68 \pm 0.156$ & $\mathrm{np}$ & $0.35 \pm 0.12$ & $\mathrm{np}$ \\
\hline NS (\%) & 32.9 & 28.6 & 4.1 & 22.1 & 10.3 & 0.0 & 2.2 & 0.0 \\
\hline $\begin{array}{l}\text { Nivel de } \\
\text { significancia }\end{array}$ & ** & ns & $* *$ & 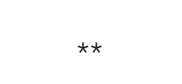 & ** & ns & ** & ns \\
\hline
\end{tabular}

Datos son promedio de los 30 genotipos \pm error estándar de la media. ** Significativamente diferentes (P $\leq 0.01)$. ns: no significativo. M: mesófilo; CB: células buliformes; F: floema; VH: vaina del haz vascular; E: epidermis; EVH no-l: extensión no lignificada de la vaina del haz vascular; X: xilema; EVH-l: extensión lignificada de la vaina del haz vascular. 


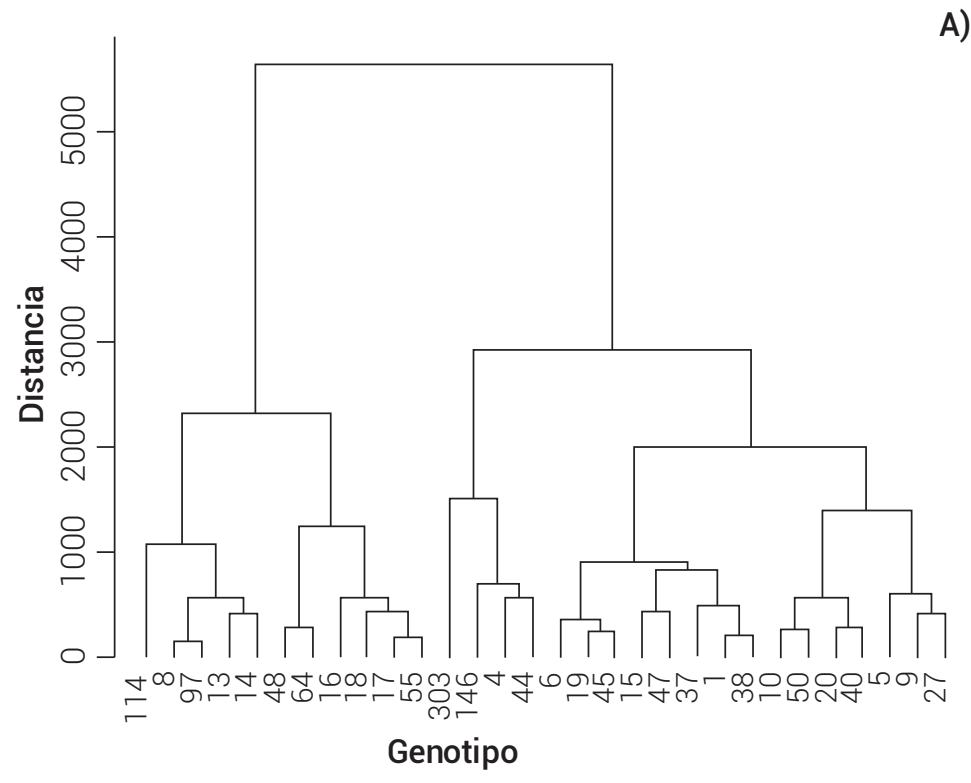

A)

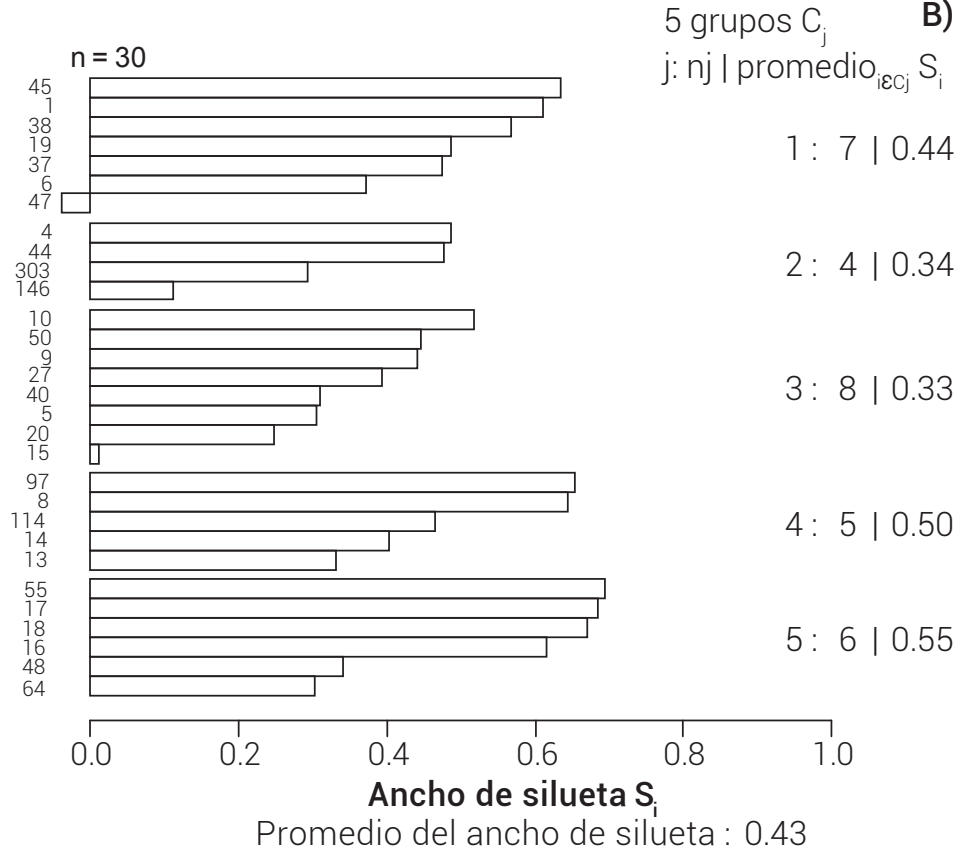

Figura 3. Agrupamiento jerárquico de genotipos de Bouteloua curtipendula para tejido altamente digestible (A) y gráfica de los promedios del ancho de silueta para cada agrupamiento (B).

reducir el efecto ambiental, por lo que la variación en el área de los tejidos se debe a atributos conservados. Se ha demostrado que los tamaños de las células buliformes están relacionados con la disponibilidad de agua; por ejemplo, en Bouteloa repens, las mayores variaciones en células buliformes fueron observadas en la época de sequía (21.8\%) y la época de lluvias (18.3\%), pero el mesófilo y la vaina del haz no presentaron variación (Chamorro et al., 2005b). Lo anterior sugiere que el tamaño de las células buliformes puede ser un atributo no elegible para estudios de selección

El agrupamiento de TPD mostró diferencias entre grupos $(P \leq 0.05)$, donde el grupo 5 mostró los mayores valores de epidermis (9.8\%) con respecto al total del área foliar. En el pasto tropical $\mathrm{C}_{4}$ Botriochloa pertusa la epidermis representó $12 \%$, mientras que en $B$. repens este tejido representó el $15 \%$, y esta diferencia fue atribuida 
al genotipo (Campos et al., 2002; Chamorro et al., 2005a; Wilson et al., 1983).

La extensión de la vaina del haz vascular no lignificada (EVH-no-I) fue observada tanto en NP como en NS, y mostró variación en el grado de lignificación entre genotipos. El G15 mostró la mayor área $\left(1.07 \pm 0.21 \mu \mathrm{m}^{2} \times 10^{3}\right)$ y fue diferente $(P \leq 0.05)$ al resto de los demás genotipos. La digestibilidad de este tejido es dependiente de la edad y la especie, con una gran variación entre especies (Buxton y Redfearn, 1997; Wilson y Mertens 1995). Los T no-D (X y $\mathrm{EVH}-\mathrm{I}$ ) representaron en promedio $9.4 \%$ del total de la hoja de B. curtipendula. La EVH-I solamente fue observada en las NP y el $X$ fue nueve veces mayor en NP que en NS.

Con respecto al área foliar total, los genotipos G16, G18 y G55 mostraron los mayores valores $\left(29.01 \pm 3.32 \times 10^{3} \mu^{2}\right.$, $27.33 \pm 3.0 \times 10^{3} \mu m^{2}$ y $\left.26.75 \pm 1.73 \times 10^{3} \mu m^{2}\right)$ (Figura 4). El

Cuadro 3. Promedios de áreas correspondientes a tejidos altamente digestibles (TAD), tejidos parcialmente digestibles (TPD), y tejidos no digestibles (T no-D) conforme al análisis de agrupamiento, para los atributos histológicos asociados con la digestibilidad de Bouteloua curtipendula. Los datos son promedio de $\mathbf{3 0}$ genotipos.

\begin{tabular}{lllllcccc}
\hline \multirow{2}{*}{ Grupos } & \multicolumn{3}{c}{$\begin{array}{c}\text { Tejidos altamente digestibles } \\
\left(\mu \mathrm{m}^{2} \times 10^{3}\right)\end{array}$} & \multicolumn{2}{c}{$\begin{array}{c}\text { Tejidos parcialmente digestibles } \\
\left(\mu \mathrm{m}^{2} \times 10^{3}\right)\end{array}$} & $\begin{array}{c}\text { Tejidos no digestibles } \\
\left(\mu \mathrm{m}^{2} \times 10^{3}\right)\end{array}$ \\
\cline { 2 - 9 } & $\mathrm{M}$ & $\mathrm{CB}$ & $\mathrm{F}$ & $\mathrm{VH}$ & $\mathrm{E}$ & $\mathrm{EVH} \mathrm{no-I}$ & $\mathrm{X}$ & $\mathrm{EVH}-\mathrm{I}$ \\
\hline 1 & $5.87 \mathrm{~b}$ & $4.85 \mathrm{~b}$ & $1.12 \mathrm{~b}$ & $4.11 \mathrm{~b}$ & $2.19 \mathrm{~b}$ & $0.65 \mathrm{a}$ & $1.71 \mathrm{ab}$ & $0.37 \mathrm{a}$ \\
2 & $3.92 \mathrm{~d}$ & $3.71 \mathrm{c}$ & $0.76 \mathrm{c}$ & $3.21 \mathrm{c}$ & $1.40 \mathrm{~d}$ & $0.49 \mathrm{a}$ & $1.36 \mathrm{~b}$ & $0.38 \mathrm{a}$ \\
3 & $5.05 \mathrm{c}$ & $4.16 \mathrm{c}$ & $1.05 \mathrm{bc}$ & $3.81 \mathrm{~b}$ & $1.89 \mathrm{c}$ & $0.58 \mathrm{a}$ & $1.89 \mathrm{ab}$ & $0.47 \mathrm{a}$ \\
4 & $7.03 \mathrm{a}$ & $5.09 \mathrm{~b}$ & $1.42 \mathrm{a}$ & $4.84 \mathrm{a}$ & $2.21 \mathrm{~b}$ & $0.66 \mathrm{a}$ & $2.30 \mathrm{a}$ & $0.57 \mathrm{a}$ \\
5 & $7.54 \mathrm{a}$ & $6.46 \mathrm{a}$ & $1.54 \mathrm{a}$ & $4.97 \mathrm{a}$ & $2.57 \mathrm{a}$ & $0.69 \mathrm{a}$ & $2.70 \mathrm{a}$ & $0.56 \mathrm{a}$ \\
DSH $_{0.05}$ & 0.65 & 0.72 & 0.28 & 0.56 & 0.28 & 0.27 & 0.81 & 0.24 \\
Nivel de $_{\text {significancia }}$ & $* *$ & $* *$ & $* *$ & $* *$ & $* *$ & $\mathrm{~ns}$ & $*$ & $\mathrm{~ns}$ \\
\hline
\end{tabular}

Letras distintas en columnas son estadísticamente diferentes ( $\mathrm{P} \leq 0.05)$; ns: no significativo; M: mesófilo, CB: células buliformes; F: floema; VH: vaina del haz vascular; E: epidermis; EVH no-I: extensión no lignificada de la vaina del haz vascular; X: xilema; EVH-l: extensión lignificada de la vaina del haz vascular; DSH: diferencia significativa honesta.

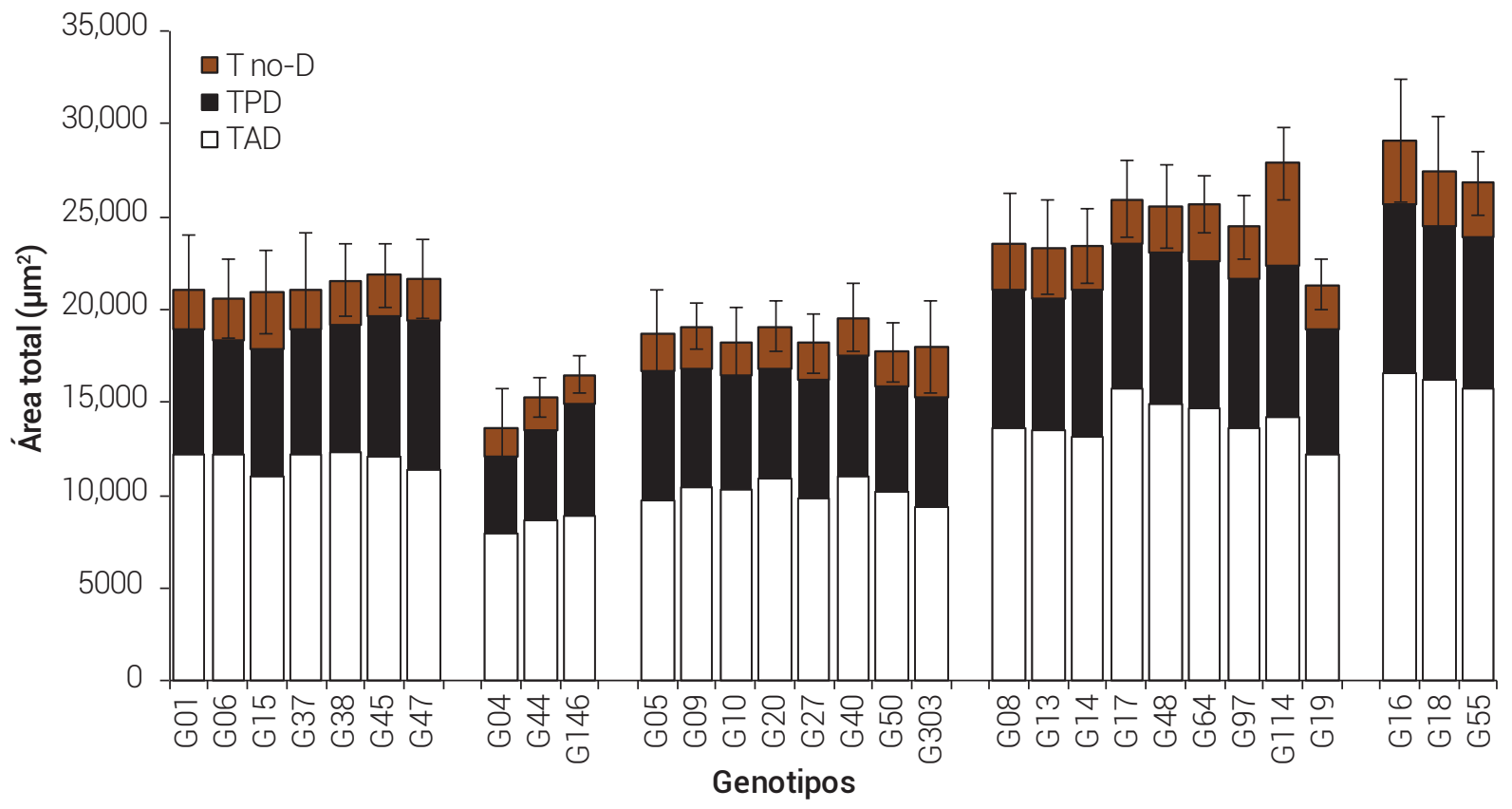

Figura 4. Contribución de los tejidos altamente digestibles (TAD), parcialmente digestibles (TPD) y no digestibles (T no-D) en el área foliar total de Bouteloa curtipendula en un agrupamiento jerárquico por área total. Los datos son promedios \pm error estándar de la media $(n=18)$. 


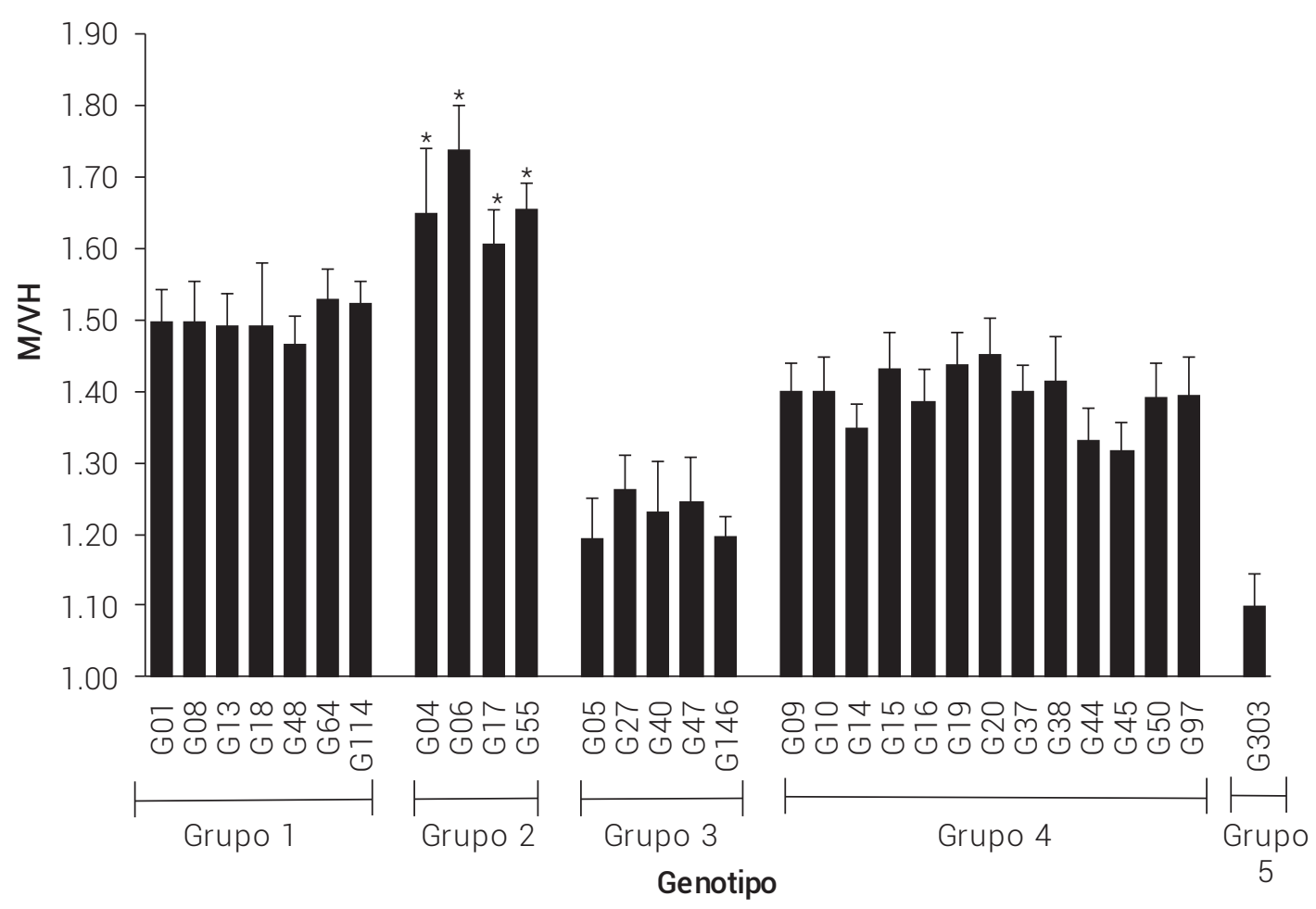

Figura 5. Relación área del mesófilo/área de la vaina del haz vascular (M/VH) de 30 genotipos de Bouteloa curtipendula en un agrupamiento jerárquico. Los datos son promedios \pm error estándar de la media $(n=18)$.

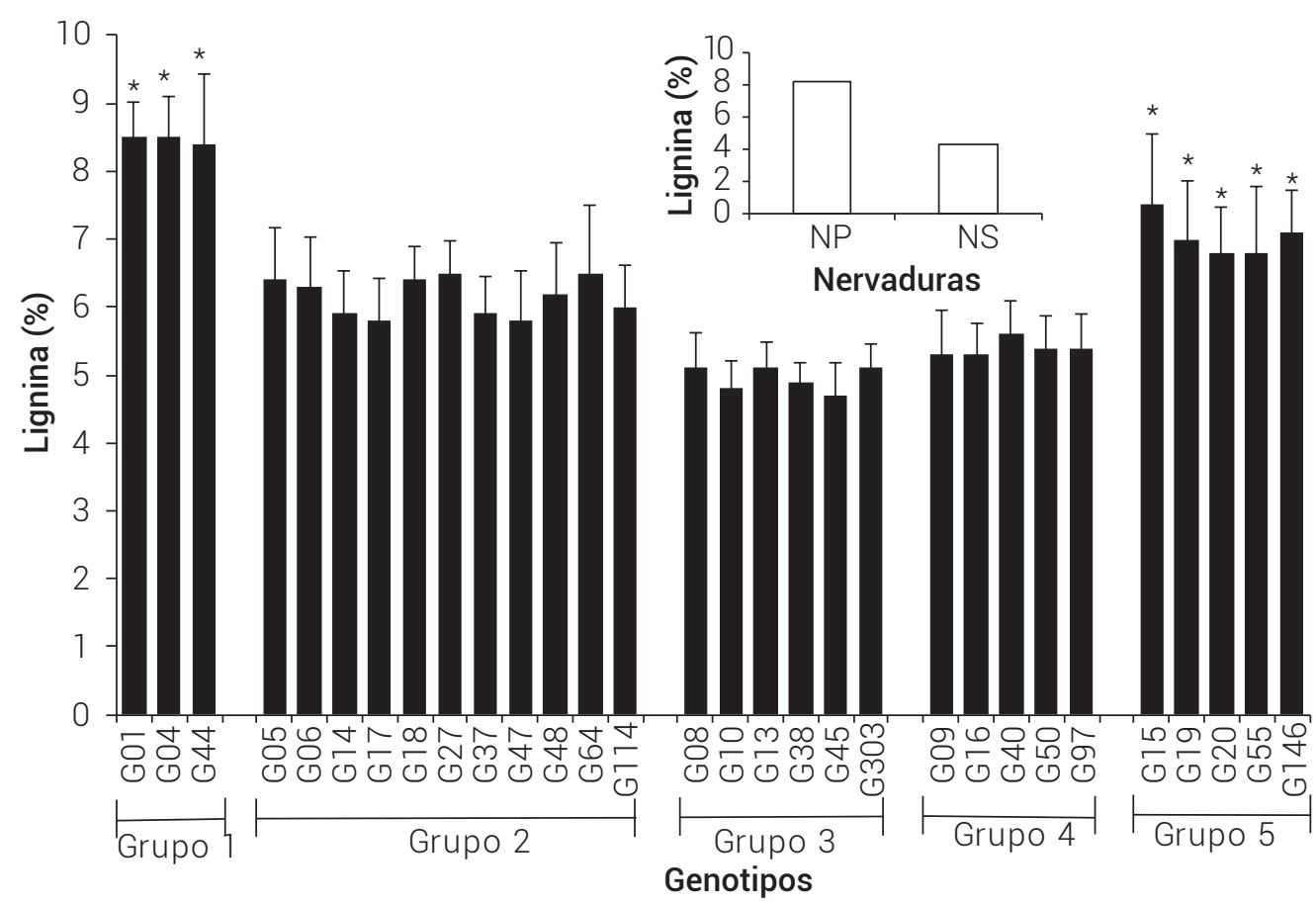

Figura 6. Área relativa de tejido lignificado en 30 genotipos de Bouteloa curtipendula, en agrupamiento jerárquico. Datos son promedios \pm error estándar de la media. NP. nervadura primaria; NS: nervadura secundaria. 
mesófilo fue el tejido con mayor área (28 \%), la EVH aportó $20 \%$ y el X contribuyó con $8.9 \%$. La relación M/VH presentó diferencias significativas $(P \leq 0.01)$ entre grupos. Los datos del presente estudio revelaron que los genotipos $\mathrm{G} 06$ y G55 presentaron los valores más altos de la razón M/ VH (1.74 y 1.66) (Figura 5), lo que indica mayor proporción de mesofilo y baja tolerancia a sequía; en contraste, G303 tuvo el menor valor (1.1) lo que indica, según McKown y Dengler (2007) y Carmo-Silva et al. (2009), una mejora en la eficiencia hidráulica de la hoja debido a una mayor proporción de tejido vascular y su adaptación a la sequía.

\section{Tejido lignificado}

La mayor agregación de lignina ocurrió principalmente en el xilema, vaina del haz y extensión de la vaina del haz. En promedio, los 30 genotipos presentaron $6.2 \%$ de su área foliar lignificada. Las NS (4.3\%) presentaron cerca de la mitad de tejido lignificado en relación con las NP (8.2\%). Este atributo histológico mostró diferencias significativas $(P \leq 0.01$ ) entre genotipos (Figura 6). Los genotipos G10 y G45 presentaron las menores áreas lignificadas (4.8 y $4.7 \%$ ), y su contenido de lignina fue similar a Pennisetum purpureum a los $65 \mathrm{~d}$ de rebrote, pero a los $140 \mathrm{~d}$ su contenido total de lignina se incrementó hasta $8.5 \%$ (Valenciaga, 2009), donde también se ve afectada la proporción de siringil:guaiacil (Chen et al., 2002).

\section{CONCLUSIONES}

Existe variabilidad en los atributos histológicos asociados a la digestibilidad y a la agregación de lignina, lo que permitió formar grupos de B. curtipendula. Las diferencias entre áreas de tejidos para NP y NS está estrechamente relacionada con la cantidad de tejidos digestibles, pero no con los porcentajes de lignina observados. Los genotipos G55, G17, G18, G16, G48, G64 formaron un grupo con las mayores áreas de TAD; G55 se agrupó con los genotipos de mayor proporción de $\mathrm{M} / \mathrm{VH}$, pero en contraste, también se agrupó con los genotipos de mayor proporción de tejido con lignina. Los atributos histológicos asociados a la digestibilidad permitieron seleccionar los genotipos con mejores atributos histológicos y de potencial forrajero. Los genotipos G16, G18 y G55 presentaron los mejores atributos relacionados con degradabilidad, con una mayor área de TAD. Este es el primer reporte donde se usan atributos histológicos en la selección de recursos genéticos mexicanos con potencial forrajero.

\section{AGRADECIMIENTOS}

Al CONACYT por la beca de doctorado otorgada al primer autor. Por el apoyo parcial otorgado por el proyecto 248252 de la convocatoria de Problemas Nacionales 2014. A la
LPI-16 "Innovación Tecnológica por el apoyo económico otorgado a una parte de esta investigación".

\section{BIBLIOGRAFÍA}

Akin D. E. and D. Burdick (1975) Percentage of tissue types in tropical and temperate grass leaf blades and degradation of tissues by rumen microorganisms. Crop Science 15:661-668.

Akin D. E. (1989) Histological and physical factors affecting digestibility of forages. Agronomy Journal 81:17-25.

Arellano-Cueto A., A. R. Quero-Carrillo, H. A. Zavaleta-Mancera, M. Silva-Luna, M. A. Cobos-Peralta y P. Pérez-Rodríguez (2017) Caracterización anatómica de hoja de recursos genéticos de Hymenachne amplexicaulis (Rudge) Nees. Revista Fitotecnia Mexicana 40:65-72.

Barkworth M. E., L. K. Anderton, K. M. Capels, S. Long and M. B. Piep (2007a) Manual of Grasses for North America. Utah State University Press. Logan, Utah. 640 p.

Barkworth, M. E. K. M. Capels, S. Long and L. K. Anderton (2007b) Flora of North America: North of Mexico. Vol. 24-25. Poaceae. Part 1 and 2. Oxford University Press. New York, N.Y. 944 p.

Buxton D. R. and D. D. Redfearn (1997) Plant limitations to fiber digestion and utilization. The Journal of Nutrition 127 Suppl:814S-818S.

Campos P. D. S., J. A. Gomide, E. A. Monteiro da S., D. S. Queiroz e C. A. Miranda G. (2002) Características anatômicas da lâmina foliar e do colmo de gramíneas forrageiras tropicais, em função do nível de inserção no perfilho, da idade e da estação de crescimento Revista Brasileira de Zootecnia 31:890-899.

Carmo-Silva A. E., A. Francisco, S. J. Powers, A. J. Keys, L. Ascensão, M. A. J. Parry and M. C. Arrabaça (2009) Grasses of different $C_{4}$ subtypes reveal leaf traits related to drought tolerance in their natural habitats: changes in structure, water potential, and amino acid content. American Journal of Botany 96:1222-1235.

Casler M. D. and R. D. Hatfield (2006) Cell wall composition of smooth bromegrass plants selected for divergent fiber concentration. Journal of Agricultural and Food Chemistry 54:8206-8211.

Chamorro D., J. E. Carulla y P. Cuesta (2005a) Degradación microbiana in situ de tejidos foliares de gramíneas y leguminosas y su relación con indicadores de calidad nutricional. Revista Corpoica 6:100-116

Chamorro D., J. E. Carulla y P. Cuesta (2005b) Relaciones entre estructura histológica y composición química en hojas de gramíneas y leguminosas. Revista Corpoica 6:83-99

Chen L., C. Auh, F. Chen, X. Cheng, H. Aljoe, R. A. Dixon and Z. Wang (2002) Lignin deposition and associated changes in anatomy, enzyme activity, gene expression and ruminal degradability in stems of tall fescue at different developmental stages. Journal of Agricultural and Food Chemistry 50:5558-5565.

Craig D., K. Sedivec, D. Tober, I. Russell and T. Faller (2001) Nutrient composition, productivity, and growth of selected warm-season grasses: Preliminary report. North Dakota State University Fargo, N.D. 11 p. Available at: http://www.ag.ndsu.edu/greenhouse/hettinger-rec/sheep/2001-and-past-sheep-day-articles/Warm-season\%20Grasses\%202001.pdf (Agosto 2, 2017).

Echenique V., S. Pessino, M. Díaz, J. P. Selva, G. Luciani, D. Zappacosta, G. Cervigni, M. Meier, I. Garbus, S. Cardone, R. Miranda y G. Spangenberg (2008) Aportes de la biotecnología al mejoramiento del pasto Ilorón (Eragrostis curvula). Revista Argentina de Producción Animal 28:147-164.

Gahan P. B. (1984) Plant Histochemistry and Cytochemistry. An Introduction. Academic Press. London, UK. 301 p.

GIMP Documentation Team (2010) GNU Image Manipulation Program User manual. Boston, Massachusetts. 653

González R. S. L., A. R. Quero C., O. Franco M., C. Ramírez A., H. M. Ortega E. y C. Trejo L. (2010) Efecto de la salinidad y la temperatura sobre el crecimiento del pasto Banderita [Bouteloua curtipendula (Michx.) Torr.]. Ciencia Ergo Sum 18:59-69.

Gould F. W. (1979) The genus Bouteloua (Poaceae). Annals of the Missouri Botanical Garden 66:348-416.

Jung H. G. and M. S. Allen (1995) Characteristics of plant cell walls affecting intake and digestibility of forages by ruminants. Journal of Animal Science 73:2774-2790. 
Kaufman L. and P. J. Rousseeuw (2005) Finding Groups in Data: An Introduction to Cluster Analysis. Wiley. New York, USA. 368 p.

Makino A. and B. Osmond (1991) Effects of nitrogen nutrition on nitrogen partitioning between chloroplasts and mitochondria in pea and wheat. Plant Physiology 96:355-362.

McKown A. D. and N. G. Dengler (2007) Key innovations in the evolution of Kranz anatomy and $\mathrm{C}_{4}$ vein pattern in Flaveria (Asteraceae). American Journal of Botany 94:382-399.

Morales-Nieto C., A. Quero-Carrillo, 0. Le-Blanc, A. Hernández-Garay, J. Pérez-Pérez y S. González-Muñoz (2006) Caracterización de la diversidad del pasto nativo Bouteloua curtipendula Michx. Torr. mediante marcadores de AFLP. Agrociencia 40:711-720.

Pitman W. D. and C. C. Jaynes (1980) Strains of blue grama and sideoats grama evaluated for the Southern Great Plains. Journal of Range Management 33:381-384.

R Core Team (2015) R: A language and environment for statistical computing. R Foundation for Statistical Computing. Vienna, Austria. https://www.R-project.org/ (Julio 16, 2017).

Ruzin S. E. (1999) Plant Microtechnique and Microscopy. Oxford University Press. Oxford, UK. $322 \mathrm{p}$

SAS Institute (2004) SAS/STAT® 9.1. User's Guide Cary, NC: SAS Institute Inc. Cary, N.C., USA. 5124 p.

Soukupová J., B. N. Rock and J. Albrechtová (2001) Comparative study of two spruce species in a polluted mountainous region. New Phytologist. 150:133-145.

Soukupová J. and J. Albrechtová (2003) Image analysis - tool for quantification of histochemical detections of phenolic compounds, lignin and peroxisases in neddles of Norway spruce. Biologia Plantarum 46:595-601.

USDA, United States Department of Agriculture (2011) 'El Reno' sideoats grama Bouteloua curtipendula (Michx.) Torr. United States Department of Agriculture. https://www.nrcs.usda.gov/Internet/FSE_PLANTMATERIALS/publications/kspmcrb10371.pdf (Agosto 2, 2017).

Valenciaga D., R. S. Herrera, E. de Oliveira S., B. Chongo y V. Torres (2009) Composición monomérica de la lignina de Pennisetum purpureum vc. Cuba CT-115 y su variación con la edad de rebrote. Revista Cubana de Ciencia Agrícola 43:315-319.

Wada S., H. Ishida, M. Izumi, K. Yoshimoto, Y. Ohsumi, T. Mae and Makino (2009) Autophagy plays a role in chloroplast degradation during senescence in individually darkened leaves. Plant Physiology 149:885-893

Warner D. A. and G. E. Edwards (1989) Effects of polyploidy on photosynthetic rates, photosynthetic enzymes, contents of DNA, chlorophyll, and sizes and numbers of photosynthetic cells in the $\mathrm{C}_{4}$ dicot Atriplex confertifolia. Plant Physiology 91:1143-1151.

Wilson J. R. and D. R. Mertens (1995) Cell wall accessibility and cell structure limitations to microbial digestion of forage. Crop Science 35:251-259.

Wilson J. R., R. H. Brown and W. R. Windham (1983) Influence of leaf anatomy on the dry matter digestibility of $\mathrm{C}_{3^{\prime}} \mathrm{C}_{4^{\prime}}$ and $\mathrm{C}_{3} / \mathrm{C}_{4}$ intermediate types of Panicum species. Crop Science 23:141-146.

Wittenberg K. M., E. C. Thomsett and N. Ames (1993) Use of histochemistry, section-to-slide digestion and image analysis for evaluation of digestibility in reed canarygrass (Phalaris arundinacea L.) genotypes. Canadian Journal of Animal Science 74:83-89.

Zavaleta-Mancera H. A., M. S. Hernández-Villarreal, J. A. Cuevas-Sánchez y E. M. Engleman (2003) Anatomía de la semilla de Cupania dentata (Sapindaceae) con énfasis en la semilla madura. Anales del Instituto de Biología, Serie Botánica 74:17-29. 